\title{
The Renin-Angiotensin-Aldosterone System (RAAS) Is One of the Effectors by Which Vascular Endothelial Growth Factor (VEGF)/Anti-VEGF Controls the Endothelial Cell Barrier
}

\author{
Yueru Li, * Zhonghao Yan, * Komal Chaudhry, ${ }^{\dagger}$ and Andrius Kazlauskas *
}

From the Departments of Ophthalmology \& Visual Sciences* and Physiology and Biophysics, ${ }^{\ddagger}$ University of Illinois at Chicago, Chicago; and the Southern Illinois University School of Medicine, ${ }^{\dagger}$ Carbondale, Illinois

\author{
Accepted for publication \\ June 1, 2020. \\ Address correspondence to \\ Andrius Kazlauskas, Ph.D., \\ University of Illinois at Chi- \\ cago, Lions Illinois Eye \\ Research Institute, 1905 W \\ Taylor St., Room 245, Chicago, \\ IL 60612. E-mail: ak20@uic \\ edu.
}

\begin{abstract}
Leakage of retinal blood vessels, which is an essential element of diabetic retinopathy, is driven by chronic elevation of vascular endothelial growth factor (VEGF). VEGF quickly relaxes the endothelial cell barrier by triggering signaling events that post-translationally modify pre-existing components of intercellular junctions. VEGF also changes expression of genes that are known to regulate barrier function. Our goal was to identify effectors by which VEGF and anti-VEGF control the endothelial cell barrier in cells that were chronically exposed to VEGF (hours instead of minutes). The duration of VEGF exposure influenced both barrier relaxation and anti-VEGF-mediated closure. Most VEGF-induced changes in gene expression were not reversed by anti-VEGF. Those that were constitute VEGF effectors that are targets of anti-VEGF. Pursuit of such candidates revealed that VEGF used multiple, nonredundant effectors to relax the barrier in cells that were chronically exposed to VEGF. One such effector was angiotensin-converting enzyme, which is a member of the renin-angiotensin-aldosterone system (RAAS). Pharmacologically antagonizing either the angiotensin-converting enzyme or the receptor for angiotensin II attenuated VEGF-mediated relaxation of the barrier. Finally, activating the RAAS reduced the efficacy of anti-VEGF. These discoveries provide a plausible mechanistic explanation for the longstanding appreciation that RAAS inhibitors are beneficial for patients with diabetic retinopathy and suggest that antagonizing the RAAS improves patients' responsiveness to anti-VEGF. (Am J Pathol 2020, 190: 1971-1981; https://doi.org/10.1016/j.ajpath.2020.06.004)
\end{abstract}

In 2013, approximately 1 of 19 people had diabetes mellitus (DM). The incidence of this life-long affliction is increasing: 1 of every 14 people are predicted to have DM in $2030 .{ }^{1}$ This metabolic disease causes both macrovascular and microvascular dysfunction, which manifests in multiple organs (eyes, kidneys, skin, etc) and systems (eg, cardiovascular, neural, etc). ${ }^{2}$ Thus, a substantial percentage of our world's population has diabetes, and their quality of life is compromised by the complications that they develop.

Approximately one-third of patients with diabetes have diabetic retinopathy (DR), ${ }^{3,4}$ which is diagnosed and staged based on the anatomy of the retinal vasculature, the thickness of both individual layers within the retina, and the total retina. High blood pressure is one of the risk factors for developing $\mathrm{DR},{ }^{5}$ and medications that control blood pressure reduce the risk of developing DR and increase the likelihood of regression., ${ }^{6,7}$ These benefits have been reported for patients taking antagonists of the reninangiotensin-aldosterone system (RAAS), which include inhibitors of angiotensin-converting enzyme (ACE) (such as ramipril and trandolapril), and angiotensin II (AII) receptor antagonists (such as valsartan and telmisartan). The key

Supported by University of Illinois at Chicago (UIC) start-up funds (A.K.), JDRF grant 2-SRA-2019-856-S-B (A.K.), Illinois Society for the Prevention of Blindness (ISPB) (Y.L.), and NIH grant UL1TR002003 (UIC Bioinformatics).

Disclosures: None declared. 
elements of the RAAS include renin-mediated cleavage of angiotensinogen to produce angiotensin $\mathrm{I}$, which ACE proteolyzes to generate the 8 amino acid AII that binds to G protein-coupled receptors (GPCRs) such as AGTR1 (angiotensin II receptor 1). ${ }^{8,9}$ Thus, RAAS antagonists, which reduce the activity of ACE- or AII-dependent activation of AGTR1, are beneficial for patients with DR.

Animal models of DR can reproduce this clinical phenomenon. Antagonizing the RAAS prevents diabetic rodents from developing DR. ${ }^{10-13}$ Systemic administration of RAAS inhibitors have no effect on DM, yet they prevent leakage of retinal blood vessels, a hallmark of DR. Animals treated in this way also have lower levels of retinal vascular endothelial growth factor (VEGF) A, which could result from not developing DR. Alternatively, antagonizing the RAAS may prevent the DM-driven rise in VEGF, which promotes DR. The underlying mechanism by which RAAS inhibitors protect experimental animals from DR and are beneficial in patients has not been addressed.

Neutralizing retinal VEGF is one of the ways that patients with DR are treated. Although many patients with DR benefit from anti-VEGF, the response is not uniform. ${ }^{14-17} \mathrm{~A}$ recent report, which indicates that genetic variants in the RAAS predict response to anti-VEGF in cancer patients, ${ }^{18}$ raises the intriguing question of whether consumption of RAAS antagonists affects the efficacy of anti-VEGF administered to patients with DR.

In this study, how VEGF/anti-VEGF controlled permeability of primary human retinal endothelial cells that were enduringly exposed to VEGF was investigated. The authors discovered that VEGF increased, and anti-VEGF decreased, the expression of $A C E$, a driver of the RAAS. Antagonists of the RAAS partially suppressed VEGF-mediated relaxation of the barrier indicating that VEGF uses multiple effectors to relax the barrier, and that the RAAS is one of them. Finally, enforced activation of RAAS attenuated antiVEGF's ability to reclose the barrier. These results provide mechanistic insights regarding the relationship between RAAS and VEGF/anti-VEGF-dependent control of the endothelial cell barrier, which are relevant to current management of patients with DR.

\section{Materials and Methods}

\section{Materials}

Human retinal endothelial cells (HRECs) were purchased from Cell Systems (ACBRI 181; Kirkland, WA). They were derived from donor A, a 26-year-old Caucasian male. Lonza endothelial cell basal medium-2 (EBM-2, CC3156) and Lonza SingleQuots endothelial cell growth medium-2MV (EGM$2 \mathrm{MV}, \mathrm{CC} 4147)$ for tissue culture was procured from Lonza Bioscience (Verviers, Belgium). D-(+)-glucose (G7021), recombinant human ACE (SAE0075), recombinant human AII (A9525), ramipril (ACE inhibitor, R0404), trandolapril (ACE inhibitor, T4827), valsartan (AGTR1 inhibitor, SML0142), telmisartan (AGTR1 inhibitor, T8949), fluorescein isothiocyanate (FITC)-dextran (70 KDa), and Celite 545 (22140) were purchased from Sigma-Aldrich (St. Louis, MO). Recombinant human IL-1 $\beta$ (200-01B) was purchased from PeproTech, Inc. (Rocky Hill, NJ). Recombinant human VEGF-A (VEGF 165; 293-VE) and human tumor necrosis factor $\alpha$ (TNF- $\alpha$ ) protein (210-TA) were purchased from R\&D Systems (Minneapolis, MN). Aflibercept (Eylea) and bevacizumab (Avastin) were from Regeneron Pharmaceuticals, Inc. (Tarrytown, NY) and Genentech, Inc. (South San Francisco, CA), respectively. RNeasy Plus Mini Kit used for RNA isolation was obtained from QIAGEN (Hilden, Germany). The High Capacity cDNA Reverse Transcription Kit used for cDNA synthesis and Fast SYBR Green Master Mix used for real-time PCR were purchased from Applied Biosystems (Thermo Fisher Scientific, Waltham, MA). The Transwell 24-Well Permeable Support System used for FITC-dextran permeability assay was purchased from Corning, Inc. (3391; Corning, NY). Disposable Electrode Arrays $(8 \mathrm{~W} 10 \mathrm{E}+\mathrm{PC})$ used in transendothelial electrical resistance (TEER) assay for measuring cellular permeability were purchased from ECIS Cultureware (Applied BioPhysics, Inc., Troy, NY). Palmitic acid (N-16-A) was from Nu-Chek Prep, Inc. ( Elysian, MN).

\section{Culture of Human Retinal Endothelial Cells}

Primary HRECs (Cell Systems, Kirkland, WA) were used to establish an in vitro model of DR. HRECs were cultured in complete Lonza medium (EBM-2 supplemented with the EGM-2MV SingleQuots kit). High-glucose-treated HRECs were generated by culturing cells in complete Lonza medium containing $30 \mathrm{mmol} / \mathrm{L}$ D-glucose for at least 10 days; medium was changed every 24 hours. The second model of DM was generated by exposing high-glucose-treated cells to palmitate $(50 \mu \mathrm{mol} / \mathrm{L})$ and tumor necrosis factor alpha $(\mathrm{TNF} \alpha)(0.1$ $\mathrm{ng} / \mathrm{mL}$ ) for 24 hours prior to the start of the experiment.

Palmitate was added to complete Lonza medium by first loading it with bovine serum albumin as described by Spector and Hoak. ${ }^{19}$ Briefly, palmitic acid dissolved in hexanes was coated onto Celite and then the hexanes were evaporated under nitrogen. Complete Lonza medium containing sufficient bovine serum albumin to achieve a 5:1 molar ratio was then added (palmitic acid:bovine serum albumin) and the solution was incubated for 30 minutes at room temperature. The Celite was removed by centrifugation, and the $\mathrm{pH}$ of the supernatant was adjusted to 7.4, filtered through a $0.22-\mu \mathrm{m}$ filter, and then frozen until use.

\section{Library Preparation and RNASeq}

RNAseq libraries of poly (A) RNA from $500 \mathrm{ng}$ of total RNA obtained from HRECs treated with vehicle, VEGF, and VEGF + anti-VEGF were generated using the Clontech SMARTer Stranded RNA-Seq Kit (634838; Clontech Laboratories, Mountain View, CA) for Illumina HiSEQ4000 (single end 50p; Illumina, San Diego, CA). Fastq were 
generated using Illumina-provided demultiplexing software, bcl2fastq2 Conversion Software version 2.20.

\section{RNAseq $Q C$ and Quantification}

RNAseq analysis was performed by the University of Illinois at Chicago or University of Chicago Core Facilities. Raw reads were aligned to reference genome hg38 using the BWA-MEM software package version 0.7.12. ${ }^{20}$ Genes was quantified using featureCounts ${ }^{21}$ as raw read counts. Differential expression statistics (fold-change and $P$-value) were computed using the edgeR software package version 3.14.0..$^{22,23}$ Multigroup statistics were computed using edgeR's generalized linear modeling framework, and pairwise comparisons were computed using exactTest. In all cases, $P$-values were adjusted for multiple testing using the false discovery rate correction of Benjamini and Hochberg. ${ }^{24}$ Differences in expression were considered statistically significant based on a false discovery rate threshold of 5\% (0.05). Bioinformatics analysis in the project described was performed by the University of Illinois at Chicago Research Informatics Core.

\section{Clustering and Visualization}

Distinct and robustly separable genomic patterns were identified using a discovery clustering analysis that pairs kmeans clustering with reproducibility clustering statistics. Inherent limitations in k-means clustering were overcome-which requires a priori selection of the number of clusters $\mathrm{k}$ and gives variable results with different initializations - by assessing cluster reproducibility. In particular, k-means clustering were performed with 10 random initializations on a range of cluster numbers $\mathrm{k}$ (2 to 20). For each $\mathrm{k}$, the reproducibility of the repeated clustering runs was evaluated using the Rand Index, similar to the approach outlined by Senbabaoglu and coworkers. ${ }^{25}$ The largest $\mathrm{k}$ that yields highly reproducible clusters was then chosen. Patterns from clusters were visualized using heatmaps and boxplots to aid in interpretation.

\section{Pathway Analysis}

Gene sets obtained from the hierarchical clustering were analyzed in MetaCore (Clarivate Analytics, Philadelphia, PA) using the Pathway Maps tool.

\section{PCR Analysis}

HRECs were seeded at full confluency. VEGF was added to a final concentration of $2 \mathrm{nmol} / \mathrm{L}$ and then cultured for an additional $0.5,1.5$, or 8 hours. For the anti-VEGF group, after 8 hours of VEGF incubation, aflibercept $(1 \mu \mathrm{mol} / \mathrm{L})$ was added for an additional 8 hours. The cells were lysed, mRNA was isolated and used to synthesize cDNA. Quantitative PCR was performed using the real-time PCR
HT7900 system (Applied Biosystems; Thermo Fisher Scientific). The threshold cycle $\left(\mathrm{C}_{\mathrm{T}}\right)$ of each transcript was normalized to the average $C_{T}$ of the housekeeping genes ( $\beta$-actin and GAPDH). Fold differences were determined by the $2-\Delta \Delta C_{T}$ method.

\section{Western Blot}

HRECs that were pre-incubated with vehicle or VEGF $(1 \mathrm{nmol} /$ L) for 24 hours were treated with anti-VEGF (aflibercept) for indicated time periods. The cells were then rinsed with ice-cold phosphate-buffered saline $(137 \mathrm{mmol} / \mathrm{L} \mathrm{NaCl}, 2.7 \mathrm{mmol} / \mathrm{L}$ $\mathrm{KCl}, 8 \mathrm{mmol} / \mathrm{L} \mathrm{Na}_{2} \mathrm{HPO}_{4}$, and $2 \mathrm{mmol} / \mathrm{L} \mathrm{KH}_{2} \mathrm{PO}_{4}$ ) and lysed in electrophoresis sample buffer (10 mmol/L EDTA; $4 \%$ sodium dodecyl sulfate; $5.6 \mathrm{~mol} / \mathrm{L}$ 2-mercaptoethanol; $20 \%$ glycerol; $200 \mathrm{mmol} / \mathrm{L}$ Tris- $\mathrm{HCl}, \mathrm{pH} 6.8$; and $0.2 \%$ bromophenol blue). Proteins were resolved on a 10\% SDS-polyacrylamide gel and subjected to Western blot analysis.

\section{Fluorescein Isothiocyanate (FITC)-Dextran Assay Measuring Cellular Permeability}

Cellular permeability in HRECs was measured according to the manufacturer's instruction. Specifically, HRECs were seeded at full confluency onto gelatin-coated 24-transwell inserts $(0.4-\mu \mathrm{m}$ polyester membrane; Corning). The cells were then preincubated with ACE inhibitors (ramipril, trandolapril) or AGTR1 inhibitor (valsartan) for 2 hours. After pretreatment, HRECs were stimulated with VEGF ( $2 \mathrm{nmol} / \mathrm{L}$ ) overnight, and monolayer solute permeability was determined by addition of a 70-KDa molecular weight FITC-dextran (Merck Millipore, Burlington, MA) and subsequent measurement of fluorescence intensity using a Synergy HTX microplate reader (BioTek, Winooski, VT). Fluorescence intensity was measured in duplicate per condition and normalized to untreated control cells.

\section{TEER Measuring Cellular Permeability}

Cell permeability was assessed by measuring changes in TEER using electrical cell-substrate impedance sensing (Applied Biophysics, Troy, NY). HRECs were seeded at full confluency in 8-well chamber slides equipped with gold-coated microelectrodes. The electric current passing through the endothelial monolayers was measured independently in each chamber. TEER was measured continuously and in real time before, during and after the treatment of the cells. When RAAS inhibitors (ramipril, trandolapril, valsartan, and telmisartan) were used, they were added 2 hours before addition of VEGF.

\section{Statistical Analysis}

The results are expressed as means $\pm \mathrm{SD}$. Differences among groups were evaluated by analysis of variance; statistical significance of differences between groups was assessed 

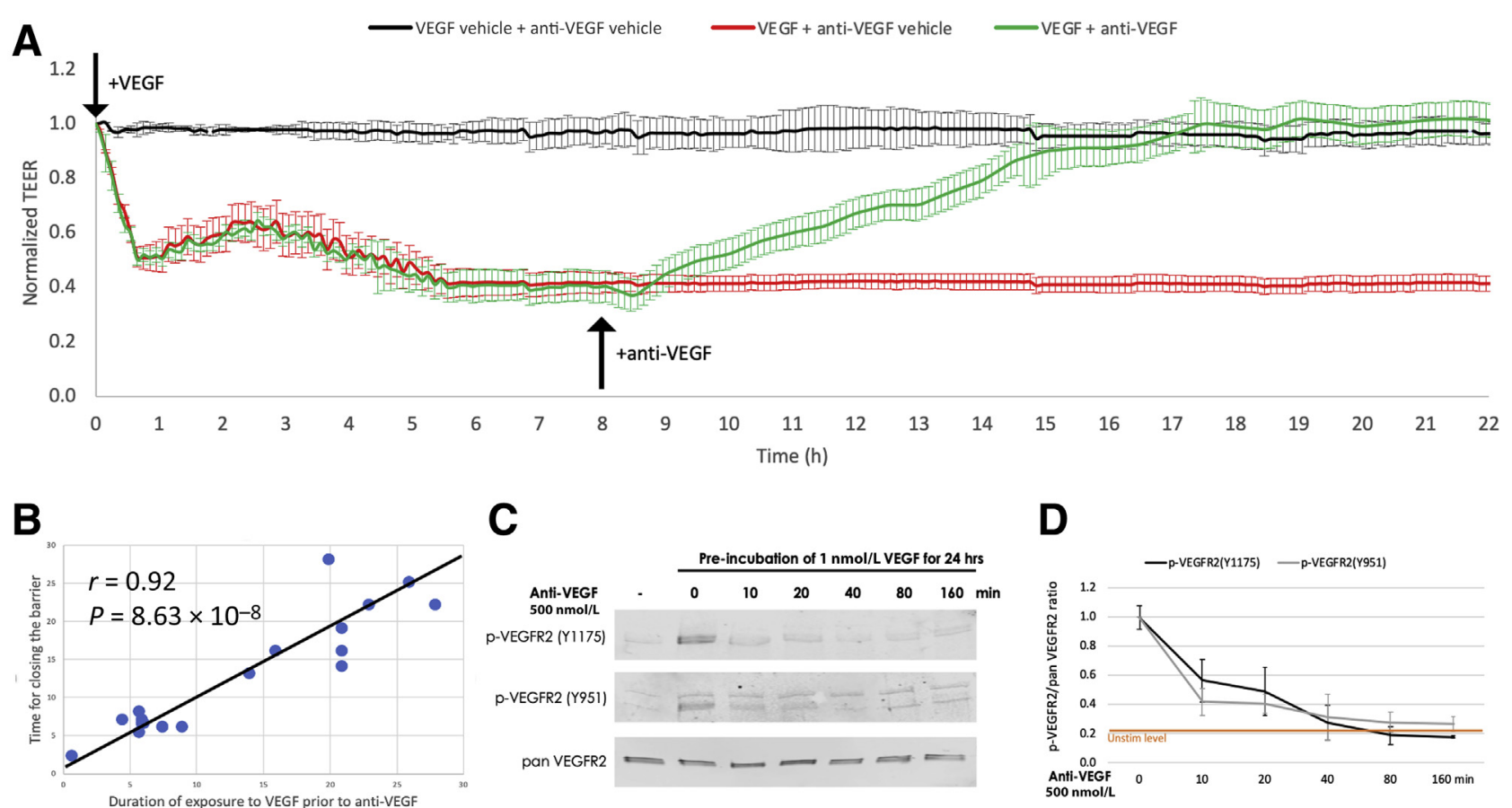

Figure 1 Signaling events did not appear to fully account for how vascular endothelial growth factor (VEGF)/anti-VEGF governed the barrier in response to prolonged exposure to VEGF. A: The transendothelial electrical resistance (TEER) of confluent monolayers of human retinal endothelial cells (HRECs) was measured for 8 hours following the addition of either VEGF vehicle or VEGF ( $1 \mathrm{nmol} / \mathrm{L})$. At the 8-hour timepoint, cells received either anti-VEGF ( $500 \mathrm{nmol} / \mathrm{L}$ aflibercept), or aflibercept buffer, and then TEER was monitored for an additional 14 hours. A high TEER value indicates effective barrier function, that is, low permeability. B: The type of experiment shown in $\mathbf{A}$ was repeated, varying the duration of exposure to VEGF prior to addition of anti-VEGF. Plotting the resulting data reveals a linear relationship between the duration of exposure to VEGF and how long it took the barrier to close after adding anti-VEGF; Pearson correlation test: $r=0.92$, $P=8.63 \times 10^{-8}$. C and D: Confluent monolayers of cells were exposed to VEGF ( $\left.1 \mathrm{nmol} / \mathrm{L}\right)$ for 24 hours, whereupon anti-VEGF ( $500 \mathrm{nmol} / \mathrm{L}$ aflibercept) was added for the indicated length of time. The cells were lysed, and total cell lysates were subjected to Western blot analysis using the indicated antibodies. A representative experiment is shown in C. The extent of vascular endothelial growth factor receptor 2 (VEGFR2) phosphorylation was quantified and normalized to the level of total VEGFR2. Data are expressed as means \pm SD (A and $\mathbf{D}) . n=3$ to 4 (A and $\mathbf{D}) ; n=3$ independent experiments (D).

using the $t$-test when indicated. Correlations between indicated variables were assessed using Pearson moment product correlation coefficients. Significance was defined as $P<0.05$. Graphs were created using Microsoft Excel 2019 (Microsoft, Redmond, WA) and R software version 3.6.0 (R Foundation for Statistical Computing, Vienna, Austria).

\section{Results}

\section{VEGF/Anti-VEGF Control Barrier Function of HRECs}

To investigate how VEGF/anti-VEGF control barrier function, electrical resistance across a monolayer of primary HRECs were monitored. Confluent, quiescent HRECs form a stable barrier (Figure 1A). Adding a saturating dose of VEGF ( 1 to $2 \mathrm{nmol} / \mathrm{L}$ ) quickly (within minutes) relaxed the barrier (Figure 1A). Following this acute barrier breakdown, barrier function was erratic for approximately 4 hours, whereupon it stabilized for at least 60 hours (Figure 1A and data not shown). Neutralizing VEGF with anti-VEGF (either aflibercept or bevacizumab) completely restored barrier function (Figure 1A and data not shown). The authors conclude that this experimental system is conducive to investigating the underlying mechanism by which VEGF relaxes the endothelial cell barrier and how anti-VEGF restores it. Furthermore, VEGF-mediated opening of the barrier proceeds through a process that takes hours to stabilize and is fully reversed by anti-VEGF.

Surprisingly, anti-VEGF further relaxed the barrier before closing it (Figure 1A). This phenomenon was observed routinely, although its magnitude was variable. Buffer alone, denatured anti-VEGF, or an equivalent amount of an unrelated protein (bovine serum albumin) did not cause it to occur (data not shown). These results indicate that it is dependent on anti-VEGF and suggests the barrier is not fully relaxed by prolonged exposure to a saturating dose of VEGF.

Varying the duration of exposure to VEGF influenced how long it took anti-VEGF to reclose the barrier. In the experiment shown (Figure 1A), it took 7 hours for the barrier to reform after adding anti-VEGF to cells that had been exposed to VEGF for 8 hours. Shortening or extending the duration of exposure to VEGF resulted in a corresponding change in how long it took to close the barrier after the addition of anti-VEGF (Figure 1B).

A simple explanation for the kinetics of antiVEGF-mediated barrier closure is that it reflects the kinetics of VEGFR2 inactivation. However, the authors found that this was not the case. Addition of anti-VEGF to cells that had 
A

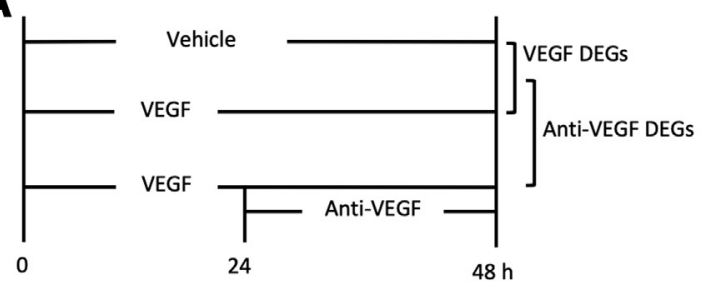

DEGs

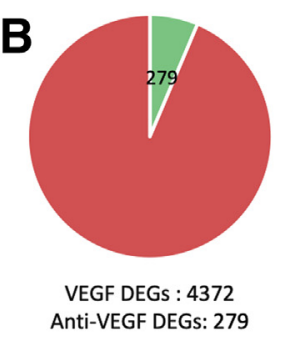

Anti-VEGF DEGs: 279

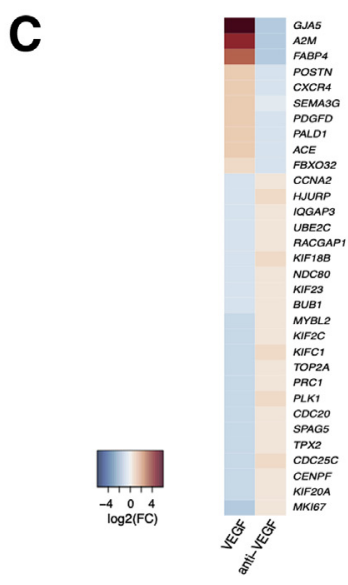

$\mathbf{E}$

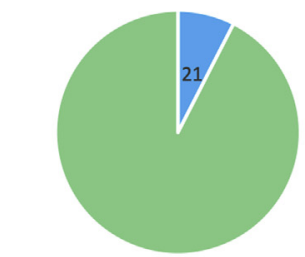

21 of the 279 anti-VEGF DEGs regulate vascular homeostasis

$\begin{array}{ll}\text { ACE } & \text { STIL } \\ \text { SELE } & \text { RBL1 } \\ \text { VCAM1 } & \text { CCNA2 } \\ \text { PDGFB } & \text { CCNB1 } \\ \text { DLL4 } & \text { GJA5 } \\ \text { EFNB2 } & \text { GJA4 } \\ \text { UNC5B } & \text { HES4 } \\ \text { INHBA } & \text { NOTCH4 } \\ \text { NRARP } & \text { CDC25C } \\ \text { HEY1 } & \text { GADD45B }\end{array}$

D

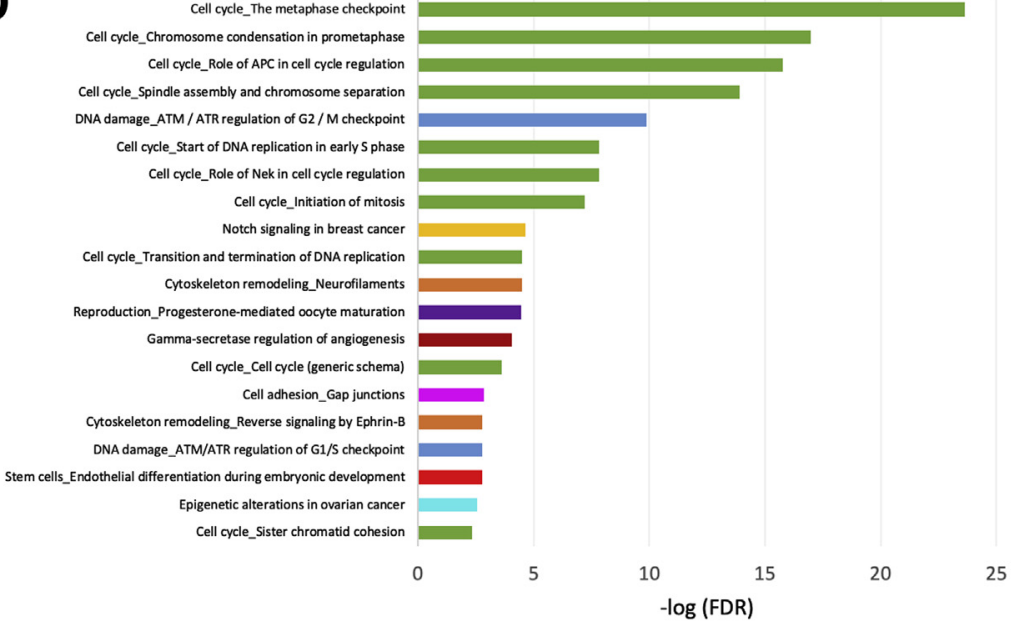

Figure 2 Vascular endothelial growth factor (VEGF) changes the expression of vascular homeostasis genes, and such changes are overcome by anti-VEGF. A: Outline of the experimental strategy. Triplicate dishes of confluent human retinal endothelial cells (HRECs) were exposed to VEGF vehicle, VEGF (1 nmol/L), or VEGF followed by anti-VEGF ( $500 \mathrm{nmol} / \mathrm{L}$ aflibercept) for the indicated duration, whereupon cells were harvested, and RNA was isolated and subjected to RNAseq analysis. Differentially expressed genes (DEGs) were identified by pairwise comparison between experimental groups as indicated in the diagram. B: The resulting RNAseq data are presented as a pie chart. VEGF induces a statistically significant $(P<0.05)$ change in expression of 4372 genes; these are VEGF DEGs. Anti-VEGF overcame the VEGF-driven change for 279 of these genes; these are the anti-VEGF DEGs. C: A heatmap of the top 32 anti-VEGF DEGs, which are arranged according to the level of fold change (FC). The expression level is indicated by color: blue is low and red is high. D: The 279 anti-VEGF DEGs were subjected to MetaCorebased pathway analysis. The top 20 pathways, sorted according to statistical significance, are presented. The colors designate a type of pathway, for instance, green is for cell cycle. E: The 21 of the 279 anti-VEGF DEGs that are known to govern vascular homeostasis are listed. FDR, false discovery rate.

been exposed to VEGF for 24 hours inactivated VEGFR2 much faster than it took to close the barrier (Figure 1, B-D) and thereby suggested the anti-VEGF-mediated barrier closure required more than simply inactivating VEGFR2.

Together, these observations support the concept that VEGF-mediated opening of the barrier is both progressive and fully reversible by anti-VEGF. Furthermore, whereas inactivating VEGFR2 is likely to initiate the process of barrier closure, these data suggest that anti-VEGF reverses additional VEGF-driven events.

\section{VEGF/Anti-VEGF-Regulated Expression of Genes that Govern Vascular Homeostasis}

Because prolonged exposure to VEGF alters expression of genes, including those that govern vascular homeostasis, it was considered whether changes in gene expression contributed to VEGF/anti-VEGF control of barrier function. To this end, the VEGF- and anti-VEGF-dependent changes in gene expression were profiled according to the experimental strategy shown (Figure 2A). The pie chart in Figure 2B illustrates the key findings of this RNAseq-based effort: VEGF altered expression of 4372 genes and antiVEGF overcame the effect of VEGF for 279 of these genes (complete and partial reversal for 162 and 117 genes, respectively). The magnitude of the VEGF- or antiVEGF-driven change in expression for the top 32 genes is shown (Figure 2C).

The 279 anti-VEGF differentially expressed genes (DEGs) were subjected to MetaCore-based pathway analysis (Figure 2D). Anti-VEGF DEGs were most strongly and commonly found to belong to the cell cycle pathway, especially as it related to the G2/M phase. Furthermore, both the identity of the genes and direction of the changes 


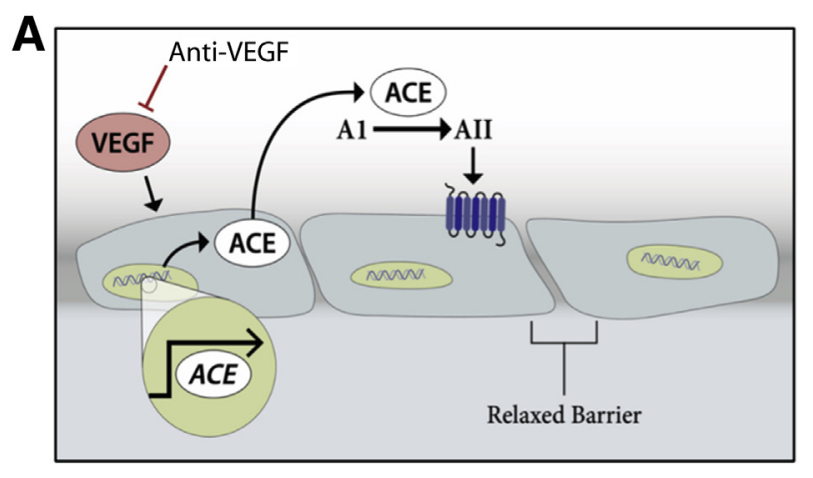

B

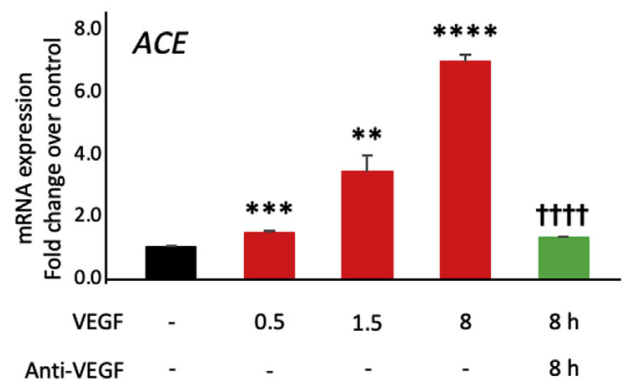

Figure 3 Temporal alignment of vascular endothelial growth factor (VEGF)/anti-VEGF-induced changes in gene expression and barrier function. A: Diagram of the working hypothesis. VEGF increases expression of angiotensin-converting enzyme $(A C E)$ and thereby converts angiotensin I (AI) into angiotensin II (AII), which binds to its receptor (encoded by the AGTR1 gene) and relaxes the barrier. By neutralizing VEGF, anti-VEGF reduces expression of $A C E$ and thereby restores barrier function. B: Triplicate dishes of cells treated as described in the legend of Figure $1 \mathrm{~A}$ were harvested, and RNA was extracted and subjected to quantitative RT-PCR using primers specific for $A C E$. The level of expression in unstimulated cells (black bar) was set to 1.0. For VEGF-treated cells (red bars), the data are expressed as fold increase over unstimulated cells. The green bars indicate the expression in cells that were first treated with VEGF and then anti-VEGF. Similar results are observed in three independent experiments. Data are expressed as means \pm SD (B). $n=3(\mathbf{B}) .{ }^{* *} P<0.01,{ }^{* *} P<0.001$, and ${ }^{* * * * P}<0.0001$ versus unstimulated cells; ${ }^{\dagger \dagger \dagger} P<0.0001$ versus antiVEGF.

suggested that acute neutralization of VEGF promotes transition of cells into G2/M. However, whereas VEGF modestly increased the percentage of the cells in $\mathrm{S}$ phase, anti-VEGF had no impact on G2/M (data not shown). This feeble effect of VEGF/anti-VEGF on the stage of cell cycle was expected for cells grown to confluence, which results in contact-dependent inhibition of cell cycle progression.

As a complementary approach to identify anti-VEGF DEGs that are necessary for anti-VEGF-mediated reclosure of the endothelial cell barrier, anti-VEGF DEGs that are known to govern vascular homeostasis were considered. To this end, a list of genes was assembled that are included in 12 Qiagen $\mathrm{RT}^{2}$ Profiler PCR Arrays: Human WNT Signaling Pathway, Human WNT Signaling Pathway Plus, Cell Junctions, Human TGFb/BMP Signaling Pathway Plus, Human TGFb/BMP Signaling Pathway, Human Notch Signaling Pathway Plus, Human Notch Signaling Pathway,
Human Hippo Signaling Pathway, Human Endothelial Cell Biology, Human Cellular Senescence, Human Angiogenesis, and Human Adherens Junctions. The following genes were added to this list because recent publications demonstrate that they contribute to vascular homeostasis: $P T P R B$, FGD5, RHOB, PIK3CD, AKT1, AKT2, AKT3, PLCG1, $M A P K 3, M A P K 1, S R C, F Y N, Y E S 1, U N C 5 B$, and $E T V 2 .{ }^{26-34}$ After eliminating the duplicates, 660 entries remained, which were designated as vascular homeostasis genes (Supplemental Table S1).

Figure 2E lists the 21 anti-VEGF DEGs that are also vascular homeostasis genes. $A C E$ was studied because the RAAS governs angiogenesis and/or endothelial cell barrier function in both experimental animals and in patients. RAAS antagonists protect diabetic animals from retinal vessel leakage. ${ }^{10-13}$ Patients taking RAAS antagonists are less likely to develop DR, and more prone to improve once they develop it. ${ }^{6,7}$ Although animal studies demonstrated a correlation between RAAS antagonism, prevention of DR, and lower levels of retinal VEGF, the underlying mechanism has not been elucidated. Furthermore, the role of RAAS in anti-VEGF-mediated barrier closure has not been addressed. As outlined below, the authors proceeded to develop and test the hypothesis that VEGF/antiVEGF-mediated control of the endothelial cell barrier involves the RAAS (Figure 3A).

In summary, protracted exposure to VEGF increased expression of many genes, including those that are involved in barrier function, and anti-VEGF overcame a subset of these changes. These results identified candidate genes by which VEGF and anti-VEGF govern barrier function in the context of prolonged exposure to VEGF.

\section{VEGF/Anti-VEGF Alters Expression of Members of the RAAS at Times Corresponding to Changes in Barrier Function}

To begin to test the hypothesis (Figure 3A), the authors investigated whether VEGF and anti-VEGF altered expression of members of the RAAS pathway at times corresponding to changes in barrier function. This was not a given because the duration of VEGF/anti-VEGF exposure in the RNAseq experiments (Figure 2A) was not the same as the TEER experiments (Figure 1A). It was observed that VEGF increased expression of $A C E$ at times corresponding to barrier relaxation, and that anti-VEGF-driven restoration of barrier function was temporally aligned with a decline in $A C E$ expression (Figures $1 \mathrm{~A}$ and $3 \mathrm{~B}$ ). Furthermore, expression of some of the other genes that were both vascular homeostasis genes and anti-VEGF DEGs did not temporally align with changes in barrier function (EFNB2, STIL, CCNA2; data not shown). In summary, ACE, a member of the RAAS is regulated by VEGF/anti-VEGF in a way and at times that support the working hypothesis (Figure 3A). 

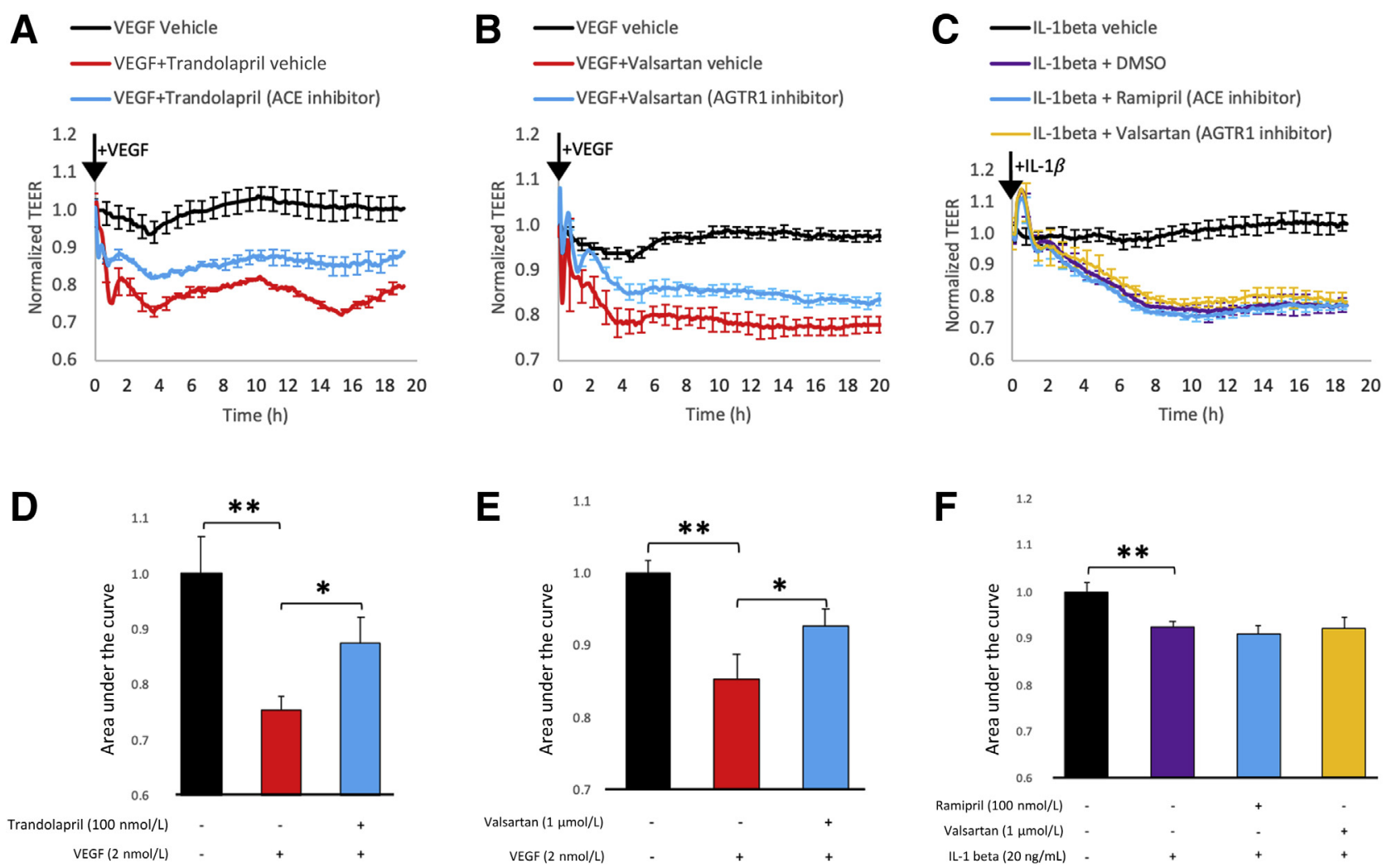

Figure 4 Antagonizing renin-angiotensin-aldosterone system (RAAS) attenuates vascular endothelial growth factor (VEGF)-induced relaxation of the endothelial barrier. Transendothelial electrical resistance (TEER) measurements of VEGF-stimulated human retinal endothelial cells (HRECS) pretreated with trandolapril (ACE inhibitor) (A and D) or valsartan (AGTR1 inhibitor) (B and E). Following a 2-hour pre-treatment with trandolapril (100 nmol/L), valsartan (1 $\mu \mathrm{mol} / \mathrm{L}$ ), or drug vehicle [dimethyl sulfoxide (DMSO)], $2 \mathrm{nmol} / \mathrm{L}$ of VEGF was added (black arrows), and TEER recorded for 20 hours. Data are expressed as a fraction of basal TEER, which was measured at the 0 time point. $\mathbf{D}$ and E: The area under the curve for the entire time course was quantified, normalized to the VEGF vehicle-treated cells. C and F: Same as panels A, B, D, and E, except that the cells were exposed to IL-1 $\beta(20 \mathrm{ng} / \mathrm{mL})$ instead of VEGF. Similar results are observed in at least three independent experiments. Data are expressed as the means \pm SD for a single representative experiment. ${ }^{*} P<0.05,{ }^{* *} P<0.01$. ACE, angiotensin I converting enzyme; AGTR1, angiotensin II receptor type 1.

\section{RAAS Is One of the Effectors by Which VEGF Relaxes the Barrier}

To test whether the RAAS participated in VEGF-mediated barrier relaxation, members of the RAAS were antagonized with inhibitors that are commonly used in patients. Trandolapril, an ACE inhibitor, reduced the responsiveness of cells to VEGF (Figure 4, A and D). Similarly, valsartan, an antagonist of the AII receptor (AGTR1) that is expressed by endothelial cells, reduced VEGF-induced permeability (Figure 4, B and E). Additional ACE (ramipril) or AGTR1 (telmisartan) antagonists also impaired VEGF-induced barrier relaxation (Supplemental Figure S1). These agents had no effect on basal permeability (data not shown). Furthermore, a FITC-dextran-based approach to measure permeability confirmed that inhibiting the RAAS attenuated VEGF-induced permeability (Supplemental Figure S2). Finally, IL-1 $\beta$-induced permeability was unaffected by ramipril or valsartan (Figure 4, C and F).

This data set shows that antagonizing RAAS has a relatively small effect on VEGF's ability to relax the barrier. Increasing or decreasing the dose of the inhibitors did not increase the magnitude of their effect (Supplemental
Figure S3, A and B). Similarly, lowering the dose of VEGF so that the barrier was only partially compromised did not improve the efficacy of the drugs (Supplemental Figure $\mathrm{S} 3, \mathrm{C}$ and D).

These data demonstrate that VEGF engages the RAAS to relax the barrier. Furthermore, because the effect of inhibiting the RAAS is partial, it appears that VEGF acts via additional effectors to relax the barrier in cells that are enduringly exposed to VEGF.

\section{Activating the RAAS Attenuates Anti-VEGF-Mediated Closure of the Barrier}

Although previous reports indicate a relationship between VEGF and RAAS, ${ }^{10-13}$ whether RAAS affects the efficacy of anti-VEGF has not been investigated. To this end, it was tested whether enforcing RAAS activity influenced antiVEGF-mediated closure of the barrier, by first relaxing the barrier with VEGF, and then adding anti-VEGF together with purified ACE or AII. It is shown that activating RAAS with purified ACE suppressed anti-VEGF's ability to reclose the barrier (Figure 5, A and B). Valsartan eliminated the effect of ACE, indicating that activation of AGTR1 was 

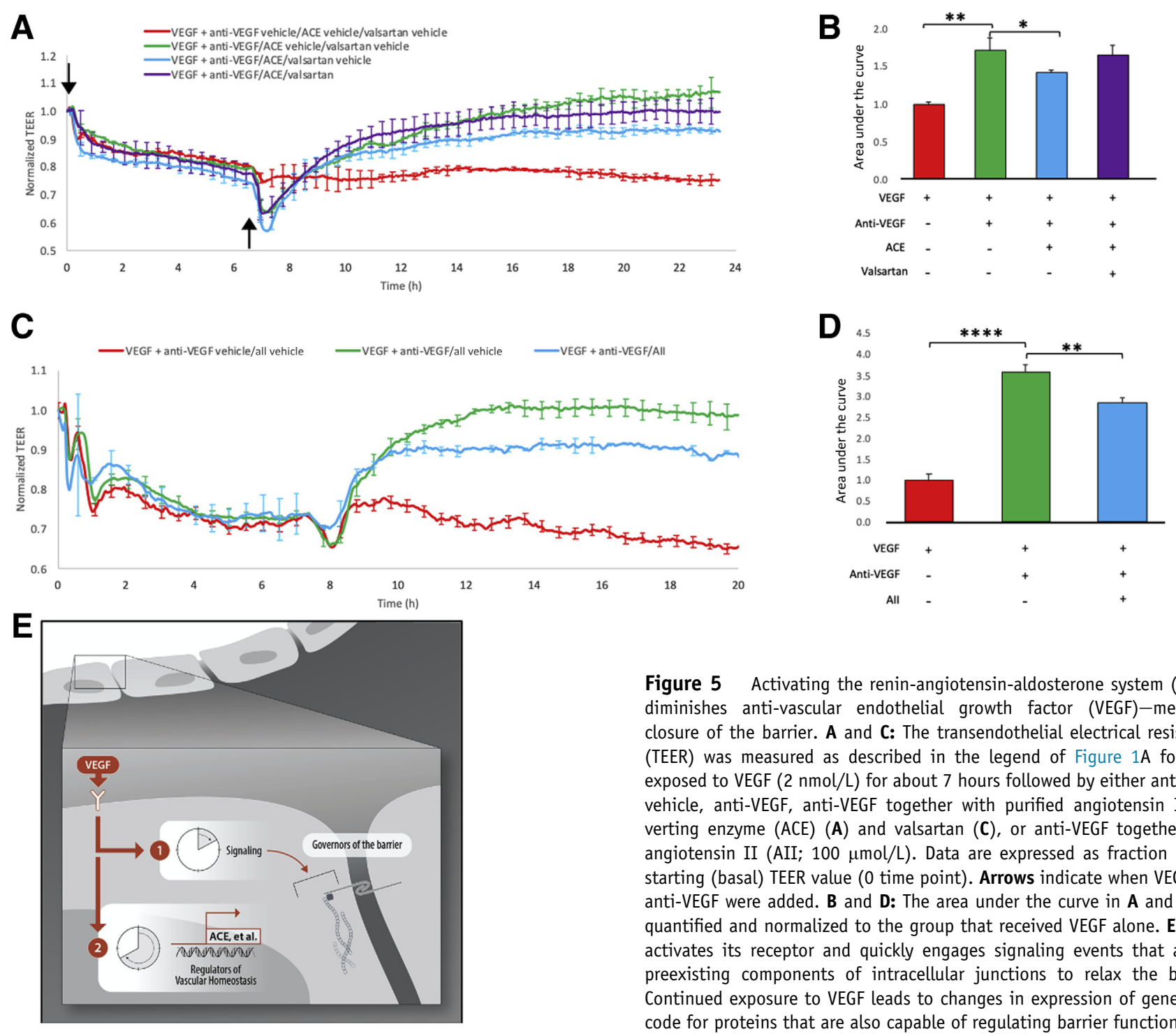

required for this phenomenon. Addition of purified AII together with anti-VEGF also attenuated anti-VEGF's ability to close the barrier (Figure 5, C and D). These data indicate that the status of the RAAS system influences antiVEGF's efficacy; an activated RAAS suppresses antiVEGF's ability to reclose the barrier.

The authors also tested whether activating the RAAS was sufficient to relax the barrier. Addition of either purified ACE or AII did not relax the barrier of cells under basal conditions (Supplemental Figure S4, A and B). A plausible reason is that AGTR1 expression, which is increased by VEGF (data not shown), was not high enough under basal conditions to enable cells to respond to the added RAAS activators.

The following experiments were performed to determine whether ACE or AII enhanced barrier relaxation in cells exposed to VEGF. Neither of the RAAS activators enhanced permeability driven by either a submaximal or
Figure 5 Activating the renin-angiotensin-aldosterone system (RAAS) diminishes anti-vascular endothelial growth factor (VEGF)-mediated closure of the barrier. A and C: The transendothelial electrical resistance (TEER) was measured as described in the legend of Figure $1 \mathrm{~A}$ for cells exposed to VEGF ( $2 \mathrm{nmol} / \mathrm{L}$ ) for about 7 hours followed by either anti-VEGF vehicle, anti-VEGF, anti-VEGF together with purified angiotensin I converting enzyme (ACE) (A) and valsartan (C), or anti-VEGF together with angiotensin II (AII; $100 \mu \mathrm{mol} / \mathrm{L})$. Data are expressed as fraction of the starting (basal) TEER value (0 time point). Arrows indicate when VEGF and anti-VEGF were added. $\mathbf{B}$ and $\mathbf{D}$ : The area under the curve in $\mathbf{A}$ and $\mathbf{C}$ was quantified and normalized to the group that received VEGF alone. E: VEGF activates its receptor and quickly engages signaling events that act on preexisting components of intracellular junctions to relax the barrier. Continued exposure to VEGF leads to changes in expression of genes that code for proteins that are also capable of regulating barrier function. Data are expressed as means \pm SD. ${ }^{*} P<0.05,{ }^{* *} P<0.01$, and $* * * * P<0.0001 . n=3$ (A-D).

maximal concentration of VEGF (Supplemental Figure S4C and data not shown). Dose response experiments indicated that $0.5 \mathrm{nmol} / \mathrm{L}$ VEGF opened the barrier to $50 \%$ of the level observed with a saturating concentration $(1 \mathrm{nmol} / \mathrm{L})$ of VEGF (data not shown). Cells make $A C E$ in response to VEGF (Figure 3), and hence, it is possible that the amount of endogenously produced ACE and AII sufficed to maximally activate the RAAS in VEGF-stimulated cells.

In conclusion, although exogenous RAAS activators did not influence basal or VEGF-driven permeability, they suppressed the ability of anti-VEGF to reclose the barrier that was opened by extended exposure to VEGF.

\section{RAAS Contributes to VEGF/Anti-VEGF Control of the Barrier in an Additional in Vitro Model}

For all of the experiments described thus far, HRECs treated with high glucose $(30 \mathrm{mmol} / \mathrm{L}$ for at least 10 days) were 
used, which is the most commonly used in vitro model of DM. ${ }^{35-38}$ Key experiments were repeated with a second in vitro model, namely one in which cells were exposed to fatty acids $(50 \mu \mathrm{mol} / \mathrm{L}$ palmitate) and cytokines $(0.1 \mathrm{ng} / \mathrm{mL}$ $\mathrm{TNF} \alpha$ ) for 24 hours prior to the start of the experiments. Similar to high glucose, free fatty acids and cytokines are elevated in patients with DM. ${ }^{39-43}$ Dose response experiments indicated that the chosen dose of palmitate and TNF $\alpha$ did not alter either basal permeability or VEGF/antiVEGF-mediated barrier relaxation/closure (Supplemental Figure S5 and data not shown). Furthermore, antagonists of the RAAS reduced VEGF-induced permeability, and RAAS activators suppressed the ability of anti-VEGF to reclose the barrier (Supplemental Figure S5). Taken together, these results demonstrate that in multiple in vitro models of DM, the RAAS is one of the effectors of VEGF and that activating the RAAS blunts the efficacy of antiVEGF.

\section{Discussion}

Using in vitro models of DM-induced endothelial dysfunction, the authors made the following discoveries:

- The duration of exposure to VEGF influences both barrier relaxation, and anti-VEGF-mediated reclosure.

- The vast majority of VEGF-induced changes in gene expression are not reversed by anti-VEGF.

- VEGF uses multiple effectors to relax the endothelial cell barrier.

- Activating the RAAS reduces the efficacy of anti-VEGF.

The duration of exposure to VEGF influences both barrier relaxation and anti-VEGF-mediated closure. These observations suggest that VEGF-driven barrier relaxation is a process that develops and matures as cells continue to be exposed to VEGF. For instance, activated VEGFR2 quickly engages signaling events that post-translationally modify existing components of adherens junctions. ${ }^{44-46}$ These early events give way to changes in expression of genes that code for proteins that can further influence barrier function via a wide spectrum of mechanisms (Figure 5E). Regulators of the extracellular matrix, the cytoskeleton, and components of the various types of junctions that connect endothelial cells all contribute to control of paracellular permeability. ${ }^{47-49}$ Thus, the nature of the VEGF-breached endothelial barrier is in part dependent on how long cells have been exposed to VEGF. Consequently, anti-VEGF's mechanism of action will depend on the duration of exposure to VEGF. In diseases such as DR, which involve chronic elevation of VEGF, the antiVEGF-mediated benefit (reduced permeability) is likely to involve not only inactivation of VEGFR2, but also change in gene expression.

By increasing production of ACE, VEGF triggers enduring permeability, which is a potential explanation for why anti-VEGF takes so long to reclose the EC barrier.
Although neutralizing VEGF occurs within minutes, it is likely to take longer for the elevated level of $A C E$ mRNA and ACE protein, AII, and AII-induced signaling to subside.

The vast majority $(94 \%)$ of VEGF-induced changes in gene expression are not reversed by anti-VEGF. This observation provides an approach to identify those VEGFregulated genes that govern barrier function, namely, those that are reversed by anti-VEGF. Furthermore, these genes are also candidate effectors of anti-VEGF. Finally, the realization that cells retain a molecular memory of VEGF (durable VEGF-induced changes in gene expression) suggest that this phenomenon may contribute to nonresponsiveness to anti-VEGF. The endothelium within blood vessels that irreversibly express genes that VEGF engages to relax the barrier would be insensitive to antiVEGF-based therapies to reverse leakage of blood vessels.

The finding that antagonizing the RAAS only partially inhibited VEGF-induced permeability indicates that VEGF engages multiple, nonredundant effectors to relax the barrier in cells that are exposed to VEGF for a prolonged time period. Identification of the RAAS as one of these effectors provides mechanistic insight into the previously reported relationship between the RAAS and VEGF. ${ }^{10-13}$

Activating the RAAS reduces the efficacy of anti-VEGF. Thus, RAAS is like cytokines or the kallikrein system, which can open the barrier independently of VEGF and hence drive leakage in the face of anti-VEGF therapy. ${ }^{50,51}$ Unlike cytokines or the kallikrein system, patients with DR are often treated with inhibitors of the RAAS. The discovery that activated RAAS blunts the efficacy of anti-VEGF predicts that anti-VEGF will provide greater benefit to patients who are consuming RAAS antagonists. This is because such antagonists work together with anti-VEGF to reclose the barrier (Figure 5), and hence, anti-VEGF constitutes the second arm of combo therapy. By contrast, anti-VEGF is simply monotherapy in patients who are not taking inhibitors of the RAAS. A clinical study comparing responsiveness to antiVEGF in patients who do and do not consume antagonists of the RAAS is an approach to address this intriguing possibility.

RAAS antagonists appear to differ in their benefit for patients with DR. A meta-analysis of patients with DR reported that ACE inhibitors reduced the risk of incidence and progression, and increased likelihood of regression, whereas AGTR1 blockers only increased the likelihood of regression. $^{7}$ In the current study's in vitro model, an obvious difference in the two types of RAAS antagonists in preventing VEGF-induced leakage was not observed. Although the strengths of this commonly-used in vitro model is its focus on endothelial cells, ${ }^{52,53}$ its weakness is the absence of other VEGF-responsive cell types. ${ }^{35-38}$ VEGFRs are expressed by multiple retinal cells types besides the endothelium, including ganglion cells, Müller cells, and amacrine cells. ${ }^{54}$ The response of these non-endothelial cell types to neutralization of VEGF may contribute to the overall anti-VEGF effect that is observed in patients. 


\section{Acknowledgments}

We thank members of the Kazlauskas lab (Anara Serikbaeva and Basma Baccouch) for their help editing the manuscript and Lauren Kalinowski for generating the diagrams.

\section{Supplemental Data}

Supplemental material for this article can be found at http://doi.org/10.1016/j.ajpath.2020.06.004.

\section{References}

1. Stitt AW, Curtis TM, Chen M, Medina RJ, McKay GJ, Jenkins A, Gardiner TA, Lyons TJ, Hammes HP, Simo R, Lois N: The progress in understanding and treatment of diabetic retinopathy. Prog Retin Eye Res 2016, 51:156-186

2. Hamilton SJ, Watts GF: Endothelial dysfunction in diabetes: pathogenesis, significance, and treatment. Rev Diabet Stud 2013, 10: $133-156$

3. Cheung N, Mitchell P, Wong TY: Diabetic retinopathy. Lancet 2010, 376:124-136

4. Yau JWY, Rogers SL, Kawasaki R, Lamoureux EL, Kowalski JW, Bek T, et al; Meta-Analysis for Eye Disease (META-EYE) Study Group: Global prevalence and major risk factors of diabetic retinopathy. Diabetes Care 2012, 35:556-564

5. UK Prospective Diabetes Study Group: Tight blood pressure control and risk of macrovascular and microvascular complications in type 2 diabetes: UKPDS 38. UK Prospective Diabetes Study Group. BMJ 1998, 317:703-713

6. Chaturvedi N, Sjolie AK, Stephenson JM, Abrahamian H, Keipes M, Castellarin A, Rogulja-Pepeonik Z, Fuller JH: Effect of lisinopril on progression of retinopathy in normotensive people with type 1 diabetes. The EUCLID Study Group. EURODIAB Controlled Trial of Lisinopril in Insulin-Dependent Diabetes Mellitus. Lancet 1998, 351: $28-31$

7. Wang B, Wang F, Zhang Y, Zhao SH, Zhao WJ, Yan SL, Wang YG: Effects of RAS inhibitors on diabetic retinopathy: a systematic review and meta-analysis. Lancet Diabetes Endocrinol 2015, 3:263-274

8. Bader M: Tissue renin-angiotensin-aldosterone systems: targets for pharmacological therapy. Annu Rev Pharmacol Toxicol 2010, 50: 439-465

9. George AJ, Thomas WG, Hannan RD: The renin-angiotensin system and cancer: old dog, new tricks. Nat Rev Cancer 2010, 10:745-759

10. Gilbert RE, Kelly DJ, Cox AJ, Wilkinson-Berka JL, Rumble JR, Osicka T, Panagiotopoulos S, Lee V, Hendrich EC, Jerums G, Cooper ME: Angiotensin converting enzyme inhibition reduces retinal overexpression of vascular endothelial growth factor and hyperpermeability in experimental diabetes. Diabetologia 2000, 43: $1360-1367$

11. Kim JH, Kim JH, Yu YS, Cho CS, Kim KW: Blockade of angiotensin II attenuates VEGF-mediated blood-retinal barrier breakdown in diabetic retinopathy. J Cereb Blood Flow Metab 2009, 29: $621-628$

12. Ebrahimian TG, Tamarat R, Clergue M, Duriez M, Levy BI, Silvestre JS: Dual effect of angiotensin-converting enzyme inhibition on angiogenesis in type 1 diabetic mice. Arterioscler Thromb Vasc Biol 2005, 25:65-70

13. Phipps JA, Clermont AC, Sinha S, Chilcote TJ, Bursell SE, Feener EP: Plasma kallikrein mediates angiotensin II type 1 receptorstimulated retinal vascular permeability. Hypertension 2009, 53: $175-181$
14. Nguyen QD, Brown DM, Marcus DM, Boyer DS, Patel S, Feiner L, Gibson A, Sy J, Rundle AC, Hopkins JJ, Rubio RG, Ehrlich JS; RISE and RIDE Research Group: Ranibizumab for diabetic macular edema: results from 2 phase III randomized trials: RISE and RIDE. Ophthalmology 2012, 119:789-801

15. Brown DM, Nguyen QD, Marcus DM, Boyer DS, Patel S, Feiner L, Schlottmann PG, Rundle AC, Zhang J, Rubio RG, Adamis AP, Ehrlich JS, Hopkins JJ; RISE and RIDE Research Group: Long-term outcomes of ranibizumab therapy for diabetic macular edema: the 36 month results from two phase III trials: RISE and RIDE. Ophthalmology 2013, 120:2013-2022

16. Heier JS, Bressler NM, Avery RL, Bakri SJ, Boyer DS, Brown DM, Dugel PU, Freund KB, Glassman AR, Kim JE, Martin DF, Pollack JS, Regillo CD, Rosenfeld PJ, Schachat AP, Wells JA 3rd; American Society of Retina Specialists Anti-VEGF for Diabetic Macular Edema Comparative Effectiveness Panel: Comparison of aflibercept, bevacizumab, and ranibizumab for treatment of diabetic macular edema: extrapolation of data to clinical practice. JAMA Ophthalmol 2016, 134:95-99

17. Diabetic Retinopathy Clinical Research Network, Wells JA, Glassman AR, Ayala AR, Jampol LM, Aiello LP, Antoszyk AN, Arnold-Bush B, Baker CW, Bressler NM, Browning DJ, Elman MJ, Ferris FL, Friedman SM, Melia M, Pieramici DJ, Sun JK, Beck RW: Aflibercept, bevacizumab, or ranibizumab for diabetic macular edema. N Engl J Med 2015, 372:1193-1203

18. Moreno-Munoz D, de la Haba-Rodriguez JR, Conde F, LopezSanchez LM, Valverde A, Hernandez V, Martinez A, Villar C, Gomez-Espana A, Porras I, Rodriguez-Ariza A, Aranda E: Genetic variants in the renin-angiotensin system predict response to bevacizumab in cancer patients. Eur J Clin Invest 2015, 45:1325-1332

19. Spector AA, Hoak JC: An improved method for the addition of longchain free fatty acid to protein solutions. Anal Biochem 1969, 32 297-302

20. Li H: Aligning sequence reads, clone sequences and assembly contigs with BWA-MEM. arXiv, 2013. arXiv 1303.3997v1 [q-bio.GN]

21. Liao Y, Smyth GK, Shi W: featureCounts: an efficient general purpose program for assigning sequence reads to genomic features. Bioinformatics 2014, 30:923-930

22. McCarthy DJ, Chen Y, Smyth GK: Differential expression analysis of multifactor RNA-Seq experiments with respect to biological variation. Nucleic Acids Res 2012, 40:4288-4297

23. Robinson MD, McCarthy DJ, Smyth GK: edgeR: a Bioconductor package for differential expression analysis of digital gene expression data. Bioinformatics 2010, 26:139-140

24. Benjamini Y, Hochberg Y: Controlling the false discovery rate: a practical and powerful approach to multiple testing. J R Stat Soc Ser B Methodol 1995, 57:289-300

25. Senbabaoglu Y, Michailidis G, Li JZ: Critical limitations of consensus clustering in class discovery. Sci Rep 2014, 4:6207

26. Dominguez MG, Hughes VC, Pan L, Simmons M, Daly C, Anderson K, Noguera-Troise I, Murphy AJ, Valenzuela DM, Davis S, Thurston G, Yancopoulos GD, Gale NW: Vascular endothelial tyrosine phosphatase (VE-PTP)-null mice undergo vasculogenesis but die embryonically because of defects in angiogenesis. Proc Natl Acad Sci U S A 2007, 104:3243-3248

27. Kurogane Y, Miyata M, Kubo Y, Nagamatsu Y, Kundu RK, Uemura A, Ishida T, Quertermous T, Hirata K, Rikitake Y: FGD5 mediates proangiogenic action of vascular endothelial growth factor in human vascular endothelial cells. Arterioscler Thromb Vasc Biol 2012, 32:988-996

28. Adini I, Rabinovitz I, Sun JF, Prendergast GC, Benjamin LE: RhoB controls Akt trafficking and stage-specific survival of endothelial cells during vascular development. Genes Dev 2003, 17:2721-2732

29. Bijli KM, Fazal F, Slavin SA, Leonard A, Grose V, Alexander WB Smrcka AV, Rahman A: Phospholipase C-epsilon signaling mediates endothelial cell inflammation and barrier disruption in acute lung injury. Am J Physiol Lung Cell Mol Physiol 2016, 311:L517-L524 
30. Ricard N, Scott RP, Booth CJ, Velazquez H, Cilfone NA, Baylon JL, Gulcher JR, Quaggin SE, Chittenden TW, Simons M: Endothelial ERK1/2 signaling maintains integrity of the quiescent endothelium. $\mathrm{J}$ Exp Med 2019, 216:1874-1890

31. Eliceiri BP, Paul R, Schwartzberg PL, Hood JD, Leng J, Cheresh DA: Selective requirement for Src kinases during VEGF-induced angiogenesis and vascular permeability. Mol Cell 1999, 4:915-924

32. Birukova AA, Shah AS, Tian Y, Gawlak G, Sarich N, Birukov KG: Selective role of vinculin in contractile mechanisms of endothelial permeability. Am J Respir Cell Mol Biol 2016, 55:476-486

33. Koch AW, Mathivet T, Larrivee B, Tong RK, Kowalski J, PibouinFragner L, Bouvree K, Stawicki S, Nicholes K, Rathore N, Scales SJ, Luis E, del Toro R, Freitas C, Breant C, Michaud A, Corvol P, Thomas JL, Wu Y, Peale F, Watts RJ, Tessier-Lavigne M, Bagri A, Eichmann A: Robo4 maintains vessel integrity and inhibits angiogenesis by interacting with UNC5B. Dev Cell 2011, 20:33-46

34. Shah AV, Birdsey GM, Randi AM: Regulation of endothelial homeostasis, vascular development and angiogenesis by the transcription factor ERG. Vasc Pharmacology 2016, 86:3-13

35. Roy S, Sala R, Cagliero E, Lorenzi M: Overexpression of fibronectin induced by diabetes or high glucose: phenomenon with a memory. Proc Natl Acad Sci U S A 1990, 87:404-408

36. Romeo G, Liu WH, Asnaghi V, Kern TS, Lorenzi M: Activation of nuclear factor-kappaB induced by diabetes and high glucose regulates a proapoptotic program in retinal pericytes. Diabetes 2002, 51: $2241-2248$

37. El-Osta A, Brasacchio D, Yao D, Pocai A, Jones PL, Roeder RG, Cooper ME, Brownlee M: Transient high glucose causes persistent epigenetic changes and altered gene expression during subsequent normoglycemia. J Exp Med 2008, 205:2409-2417

38. Chakravarthy U, Hayes RG, Stitt AW, McAuley E, Archer DB: Constitutive nitric oxide synthase expression in retinal vascular endothelial cells is suppressed by high glucose and advanced glycation end products. Diabetes 1998, 47:945-952

39. Lyons TJ, Jenkins AJ, Zheng D, Lackland DT, McGee D, Garvey WT, Klein RL: Diabetic retinopathy and serum lipoprotein subclasses in the DCCT/EDIC cohort. Invest Ophthalmol Vis Sci 2004, 45:910-918

40. Julius U: Influence of plasma free fatty acids on lipoprotein synthesis and diabetic dyslipidemia. Exp Clin Endocrinol Diabetes 2003, 111: 246-250

41. Joussen AM, Poulaki V, Mitsiades N, Kirchhof B, Koizumi K, Dohmen S, Adamis AP: Nonsteroidal anti-inflammatory drugs prevent early diabetic retinopathy via TNF-alpha suppression. FASEB J 2002, 16:438-440

42. Abu el Asrar AM, Maimone D, Morse PH, Gregory S, Reder AT: Cytokines in the vitreous of patients with proliferative diabetic retinopathy. Am J Ophthalmol 1992, 114:731-736

43. Demircan N, Safran BG, Soylu M, Ozcan AA, Sizmaz S: Determination of vitreous interleukin-1 (IL-1) and tumour necrosis factor (TNF) levels in proliferative diabetic retinopathy. Eye (Lond) 2006, 20:1366-1369

44. Dejana E: Endothelial adherens junctions: implications in the control of vascular permeability and angiogenesis. J Clin Invest 1997, 100 Suppl:S7-S10

45. Dejana E, Hirschi KK, Simons M: The molecular basis of endothelial cell plasticity. Nat Commun 2017, 8:14361

46. Claesson-Welsh L: Vascular permeability-the essentials. Ups J Med Sci 2015, 120:135-143

47. Schwartz MA, Vestweber D, Simons M: A unifying concept in vascular health and disease. Science 2018, 360:270-271

48. Augustin HG, Koh GY, Thurston G, Alitalo K: Control of vascular morphogenesis and homeostasis through the angiopoietin-Tie system. Nat Rev Mol Cell Biol 2009, 10:165-177

49. Vestweber D: VE-cadherin: the major endothelial adhesion molecule controlling cellular junctions and blood vessel formation. Arterioscler Thromb Vasc Biol 2008, 28:223-232

50. Sohn HJ, Han DH, Kim IT, Oh IK, Kim KH, Lee DY, Nam DH: Changes in aqueous concentrations of various cytokines after intravitreal triamcinolone versus bevacizumab for diabetic macular edema. Am J Ophthalmol 2011, 152:686-694

51. Gao BB, Clermont A, Rook S, Fonda SJ, Srinivasan VJ, Wojtkowski M, Fujimoto JG, Avery RL, Arrigg PG, Bursell SE, Aiello LP, Feener EP: Extracellular carbonic anhydrase mediates hemorrhagic retinal and cerebral vascular permeability through prekallikrein activation. Nat Med 2007, 13:181-188

52. Kim D, Mouritzen U, Larsen BD, Roy S: Inhibition of Cx43 gap junction uncoupling prevents high glucose-induced apoptosis and reduces excess cell monolayer permeability in retinal vascular endothelial cells. Exp Eye Res 2018, 173:85-90

53. Stewart EA, Saker S, Amoaku WM: Dexamethasone reverses the effects of high glucose on human retinal endothelial cell permeability and proliferation in vitro. Exp Eye Res 2016, 151:75-81

54. Stitt AW, Simpson DA, Boocock C, Gardiner TA, Murphy GM, Archer DB: Expression of vascular endothelial growth factor (VEGF) and its receptors is regulated in eyes with intra-ocular tumours. J Pathol 1998, 186:306-312 